\title{
PREVALÊNCIA DA ESCLEROSE MÚLTIPLA NA REGIÃO SUL: UM ESTUDO EPIDEMIOLÓGICO
}

\author{
PREVALENCE OF MULTIPLE SCLEROSIS IN THE SOUTHERN REGION: AN \\ EPIDEMIOLOGICAL STUDY
}

Natiele llucenski Marques*, Ana Vitória Pissinati Amancio, Hugo Oikawa, Millena Perin Leite, Gustavo Bacelar Peraro.

UNINGÁ - Centro Universitário Ingá, Maringá, PR, Brasil. *nilucenski@gmail.com

\section{RESUMO}

A esclerose múltipla (EM) é uma afecção neurológica desmielinizante autoimune crônica que acomete preferencialmente adultos jovens (20-40 anos), provocada por mecanismos inflamatórios e degenerativos, isso compromete a bainha de mielina que reveste os neurônios das substâncias branca e cinzenta do sistema nervoso central, comprometendo a transmissão do impulso nervoso. A evolução clínica desta patologia é subdividida em surto-remissiva, progressiva primária, progressiva secundária e surto-progressiva. Como objetivo do estudo temos a comparação dos três estados da Região Sul (RE) do país, que engloba os estados do Paraná (PR), Santa Catarina (SC) e Rio Grande do Sul (RS), nos índices de internações pela EM na faixa temporal de 2014-2019, com comparação das faixas etárias (FE) de 20-29 anos, 30-39 anos e 40-49 anos. A metodologia de desenvolvimento do Resumo foi baseada na coleta e análise de dados do Departamento de Informática do Sistema Único de Saúde (DATASUS), recorrendo ao espaço temporal de 2014-2019. Os critérios de inclusão foram os conteúdos de internações por EM, nos três estados da RE sendo eles PR, SC e RS, nas faixas etárias de 20-29 anos, 30-39 anos e 40-49 anos. Dessa forma, observa-se que a FE entre 20 a 29 anos, apresentou os menores valores percentuais (VP) em SC, os maiores no RS nos anos de 2014 e 2015, os maiores no PR em 2017, 2018 e 2019. Em 2016 apresentou valores iguais no PR e RS. A FE entre 30 a 39 anos, apresentou uma variedade maior que a FE anterior, e o RS possuiu o maior valor, exceto em 2018. A FE entre 40 a 49 anos, apresentou VP variáveis, tendo 0 RS maiores percentuais totais. Percentualmente os pacientes com EM dentre os atendimentos por Doenças do Sistema Nervoso (DN) representam de 2,26 a 3\% na Região Sul, de 1,7 a 2,82\% no PR, de 1,78 a 3,56\% em SC, de 2,95 a 3,31 no RS, no período estudado. E entre os pacientes com EM do sexo masculino o percentual para a RE variou de 27 a $32 \%$ e do sexo feminino de 68 a $73 \%$ no período determinado. Dado o exposto, percebe-se que o sexo feminino é o mais acometido na região Sul, sendo que a maioria se encontra em SC. Além disso, nota-se que a FE mais acometida no PR foi entre 40-49 anos, no entanto, a menos ocorrente nesse estado foi entre 30-39 anos, ao contrário de SC e RS, em que essa FE foi a que mais apresentou casos da doença. Porém, em todas as FE estudadas, o ano com maior prevalência de EM no PR foi 2018, sendo esse o ano com menos no RS. Já em SC, o predomínio de EM foi em 2014, mesmo ano com menos no PR. 
Ademais, entre os pacientes que foram internados por doenças do sistema nervoso, a maioria encontra-se no estado do RS, já a menor parte é do PR. Portanto, com base no valor total entre todas as idades das regiões estudadas, pode-se concluir que o estado com maior prevalência de EM é RS, seguido do PR, e, por fim, SC.

Palavras-chave: Brasil. Doenças neurológicas. Epidemiologia. Esclerose Múltipla. Prevalência. 